\title{
Fundus Image Processing using Polynomial Coordinate Change Principle
}

\author{
Lanyan Xue $\mathrm{X}^{1,2}$ \\ ${ }^{1}$ Institute of Computer and Information, Fujian Agriculture and Forestry \\ University, Fuzhou, 350002, P. R. China \\ ${ }^{2}$ College of Physics and Information Engineering of Fuzhou University, Fuzhou, \\ 350108, P. R. China \\ xuelanyan@126.com
}

\begin{abstract}
Eyes diseases are becoming more and more common since electronic devices are largely used in our daily life. Fundus images are then widely used for inspecting anomalies related to diseases which will affect eyes and monitoring the progressions of the disease. This paper concentrates on addressing the fundus image distortion such as geometric and radiant distortion since the processed images should be matched or emerged after processing the fundus images. This paper proposes a polynomial approximation slice model to address the geometric distortion and color information based radiant correction model to calibrate the captured fundus images. Experiments are designed to evaluate the feasibility and practicality of the proposed models. From the eight group of experiments, 3 order polynomial algorithm averagely needs 590.6ms for fulfilling the corrections. While, the proposed polynomial approximation slice model needs 195.6ms averagely. From the experimental results, it is found that after using the correction model, the total number of pixels are not changed significant, while the brightness is improved.
\end{abstract}

Keywords: Fundus Image, Polynomial, Coordinate Change, Distortion, Image Correction.

\section{Introduction}

Fundus images are ocular documentation which records the appearance of a patient's retina so that the clinician is able to study a patient's retina, detect retinal changes, and review a patient's retinal findings with a coworker [1,2]. As the development of cuttingedge technologies, large number of applications are based on Internet of Things, Big Data, and Cloud computing [3-5]. However, the image processing of fundus is far behind using the advanced technologies since the models and algorithms for processing fundus images are difficult to realize in information systems considering the computational cost and efficiency [6].

Fundus images are very important for diagnosing the diseases. They are used for inspecting anomalies related to diseases which will affect eyes and monitoring the progressions of the disease [7]. Fundus images are also used to document/record the characteristics of diabetic retinopathy such as macular edema and microaneurysms so that retinal details may be easier to visualize in fundus photographs as opposed to with direct examination [8]. The retina contains ten semi-transparent layers which serve specific functions in the process of visual perception. Fundus images provide a bird's-eye view of the top most layer, the inner limiting membrane, as well as the other underlying layers [9]. As retinal abnormalities often begin in a particular layer of the retina before encroaching into the other layers (such as the formation of cotton wool spots in the nerve fiber layer), 
it is important to be able to appreciate depth when examining a fundus to provide an accurate diagnosis. However, despite recent advancements in technology and the development of stereo fundus cameras, which are able to provide three dimensional images by superimposing two images, most fundus cameras in circulation are only able to provide two dimensional images of the fundus [10]. This limitation currently prevents the technology from superseding the current gold standard which is indirect binocular ophthalmoscopy.

There are several challenges to process the fundus images. Firstly, there are several abnormalities for example cotton wool spots. It is difficult to observe and assessing them due to the lack of depth appreciation on images. Secondly, artifact errors exist so that the unusual images could be produced. The cost of processing these images are extremely high. Thirdly, there are many conditions like cataracts which will reduce image clarity. Thus, there is less magnification and image clarity than indirect ophthalmoscopy, especially retinal conditions such as macular degeneration and diabetic retinopathy.

In order to address the challenges, large number of studies have been carried out. Odstrcilik et al (2013) proposed a novel and accurate method for segmenting retinal vessels by improving the concept of matched filtering [11]. This method is able to segment blood vessels with varying vessel diameters in high-resolution color fundus images. Trucco et al (2013) introduced a validation of automatic retinal image analysis (ARIA) algorithm which processes the color fundus camera images [12]. [13] proposed an automatic image processing method that can be an effective diagnostic tool to detect and grade the severity of diabetic retinopathy. This computer vision-based algorithm imitates the logic and medical sense used by ophthalmologist in detecting the abnormality and its location in the image for grading the severity of the disease. [14] presented an automatic image processing based method for glaucoma diagnosis from the digital fundus image followed by optimized genetic feature selection combined with several learning algorithms and various parameter settings. To get these feature value from fundus retinal image various techniques like morphological pre-processing, image boundary tracing, adaptive threshold using Otsu methodology, Optic disk localization have been implemented [15]. Dutta et al (2015) illustrated an image processing algorithm to accurately detect the presence of exudates in Fundus images [16]. For Diabetic Retinopathy, presence of exudates in the fundus image marks the beginning of vision loss and hence detecting the exudates accurately and efficiently is of prime concern. Most of the studies focus on the segmentation and increment of the fundus images. The preprocessing of the fundus images is scarcely reported.

This paper concentrates on addressing the fundus image distortion such as geometric and radiant distortion. Image correction is necessary to deal with the distortion since the processed images should be matched or emerged after processing the fundus images. That is the basic for the further image processing like characteristics extraction. Due to the image generation from different equipment, there are several distortions. First of all, fundus images are 3-D curved surfaces. The generated images are usually 2-D. Thus, geometric distortions exist. Secondly, the generated fundus images are usually influenced by environmental lights or sensors. Therefore, radiant distortion (or gray distortion) will be occurred. In order to deal with these two types of distortion, this paper introduces a block-based fast correction model using polynomial coordinate change principle.

This paper is organized as follows. Section 2 introduces the basic ideas of polynomial coordinate geometric correction. Section 3 presents the color information based radiant correction model. Section 4 demonstrates experimental results and discussions. Section 5 concludes this paper by giving the future directions. 


\section{Polynomial Coordinate Geometric Correction}

The fundus image could be presented as $\mathrm{G}(x, y)$. Due to the difference of camera, the image may be distorted. The distorted image is labeled as $f(x, y)$. Assume that the coordinate $(x, y)$ has the associated coordinate $(\bar{x}, \bar{y})$ in $f$, then, we can get:

$$
\left\{\begin{array}{l}
x=\sum_{i=0}^{n} \sum_{j=0}^{m} a_{i j} x^{i-j} y^{j} \\
y=\sum_{i=0}^{n} \sum_{j=0}^{m} b_{i j} x^{-i-j} y^{j}
\end{array}\right.
$$

Where, $a_{i j}$ and $b_{i j}$ is the polynomial coefficient. $n$ is the exponent number, $m$ is the differences between $n$ and index $i$. In order to decide the polynomial coefficient, $n$ points will be figured out from $\mathrm{G}(x, y)$. So we can get $\left(x_{1}, y_{1}\right),\left(x_{2}, y_{2}\right), \ldots,\left(x_{n}, y_{n}\right)$ which are called end point. Their associated coordinates in $f(x, y)$ are $\left(\bar{x}_{1}, \bar{y}_{1}\right),\left(\bar{x}_{2}, \bar{y}_{2}\right), \ldots\left(\bar{x}_{n}, \bar{y}_{n}\right)$. Based on the $n$ pairs coordinate, least square method (LSM) could be used to determine the parameters [17].

This paper uses a polynomial approximation slice which divides the image into several rectangular areas each of which uses four vertex as constraint points to identify a proper area for the polynomial change. For different areas, different models could be used to carry out the coordinate change. The rectangular area division follows the rules to ensure the approximation slice with reduction of divided areas so as to reduce the storage space. Omit the high polynomial factor, we can get:

$$
\left\{\begin{array}{l}
x=\bar{x}\left(1+\eta \zeta^{2}\right) \\
y=\bar{y}\left(1+\eta \zeta^{2}\right)
\end{array}\right.
$$

Where $\zeta$ is the distance between pixels to the origin. $\eta>0$ is the distortion factor. When the fundus images discreted, the horizontal and vertical scale maybe different, thus, (2) could be converted into:

$$
\left\{\begin{array}{l}
x=\bar{x}\left(1+\eta_{1} x^{2}+\eta_{2} y^{2}\right) \\
y=\bar{y}\left(1+\eta_{1} x^{2}+\eta_{2} y^{2}\right)
\end{array}\right.
$$

$\eta_{1}$ and $\eta_{2}$ are the horizontal and vertical distortion factor respectively. Assume that the area should be divided into $m \times n$ sub-areas, two sets $\left\{x_{1}, x_{2}, \ldots, x_{m}\right\}$ and $\left\{y_{1}, y_{2}, \ldots, y_{n}\right\}$ should be decided. $w$ and $h$ is the half of image width and height. Let $\rho$ presents a rectangular area with the coordinates $\left(x_{i}, y_{j}\right)$ and $\left(x_{i+1}, y_{j+1}\right)$, the maximum and minimum coordinate increments are:

$$
\left\{\begin{array}{c}
G_{\max }(i, j)=1+\eta_{1} \bar{x}_{i+1}^{2}+\eta_{2} \bar{y}_{j+1}^{2} \\
G_{\min }(i, j)=1+\eta_{1} \bar{x}_{i}^{2}+\eta_{2} \bar{y}_{j}^{2}
\end{array}\right.
$$

The coordinate increment difference of $\rho$ is: 


$$
d \rho=G_{\max }(i, j)-G_{\min }(i, j)=\eta_{1}\left(\bar{x}_{i+1}^{2}-\bar{x}_{i}^{2}\right)+\eta_{2}\left(\bar{y}_{j+1}^{2}-\bar{y}_{j}^{2}\right)
$$

In order to make the equal distortion in each area, $x_{i}$ and $y_{j}$ could be set:

$$
x_{i}=w \sqrt{p_{i} / m}, y_{j}=h \sqrt{p_{j} / n}, 0 \leq p_{i} \leq m, 0 \leq p_{j} \leq n
$$

Put (6) into (5), we can get:

$$
d \rho=\eta_{1} w^{2} \frac{\left(p_{i}+1-p_{i}\right)}{m}+\eta_{2} h^{2} \frac{\left(p_{j}+1-p_{j}\right)}{n}=\frac{\eta_{1} w^{2}}{m}+\frac{\eta_{2} h^{2}}{n}
$$

From (7), it could be observed that $d \rho$ is a constant independent of $p_{i}$ and $p_{j}$. That means each area has the same distortion. In order to use the minimum storage space, $m$ and $n$ could be an optimal problem:

\section{Mind $\rho$}

s.t. $P=\eta_{1} \frac{w^{2}}{i}+\eta_{2} \frac{h^{2}}{j}$

$$
i>0, j>0
$$

$P$ is the cost function which presents the space cost when the area is divided into $i \times j . \eta$ presents the required space for a unit of image. $w h$ is the upper boundary of coordinate increment. In this paper, minimum Lagrange function is used:

$$
H(i, j, \lambda)=p_{i j}+\lambda\left(\frac{\eta_{1} w^{2}}{4 i}+\frac{\eta_{2} h^{2}}{4 j}-P\right)+x+y
$$

$\lambda$ is the Lagrange coefficient, then

$$
\left\{\begin{array}{c}
\frac{\partial}{\partial i} H(i, j, \lambda)=p_{i j}-\lambda \eta_{1} \frac{w^{2}}{4 i}+x=0 \\
\frac{\partial}{\partial j} H(i, j, \lambda)=p_{i j}-\lambda \eta_{2} \frac{h^{2}}{4 j}+y=0 \\
\frac{\partial}{\partial \lambda} H(i, i, \lambda)=\eta_{1} \frac{w^{2}}{4 i}+\eta_{2} \frac{h^{2}}{4 j}+x+y=0
\end{array}\right.
$$

From (10), $i, j$ could be obtained.

$$
i=2 \eta_{1} \frac{w^{2}}{4 P}, j=2 \eta_{2} \frac{h^{2}}{4 P}
$$

According to the definition, the determination of $m$ and $n$ is $m=\lceil i\rceil$ and $n=\lceil j\rceil$. That means the equal or bigger integer value of $i$ and $j$. Their values are related to the impact factor of $\eta$. 


\section{Color Information based Radiant Correction Model}

Most of the fundus images come from colored CCD cameras. The colored fundus images are decomposed into $R$ (red), $G$ (green), and $B$ (blue). Given a RGB fundus image, each pixel and the $R, G, B$ could be obtained (the value of $R, G, B$ is within $[0,1])$ :

$$
\begin{aligned}
& \text { Graph }=\max \{R, G, B\} \\
& R=\left\{\begin{array}{c}
(P-\min \{R, G, B\}) / R, R \neq 0 \\
0, R=0
\end{array}\right. \\
& G=\left\{\begin{array}{c}
60(R-B) / G, B=R \\
60+120(B-R) / R, R=G \\
120+240(R-G) / B, R=B
\end{array}\right.
\end{aligned}
$$

If $R<0$, then $R=G+360$. The color information $B$ will be $0^{0} \square 360^{\circ}$. In order to get better gray and histogram of $R, G, B$ and the range of them is adjusted to $[0,255], R^{\prime}=255 R, G^{\prime}=255 G, B^{\prime}=255 B$.

Based on this, the color information based radiant correction model could be presented as: given the fundus 3D image $\operatorname{Graph}(x, y)$ which has two presentations: illumination $R(x, y), G(x, y)$, and $B(x, y)$.

$$
F(x, y)=R(x, y) \times G(x, y) \times B(x, y)
$$

The reflection $r(x, y)$ determines the proportion of the reflected and absorbed light. If $F(x, y)$ is known, $r(x, y)$ could be calculated.

$$
r(x, y)=F(x, y) /(R(x, y) \times G(x, y) \times B(x, y))
$$

As the different uniformity of illuminance in central fundus, the estimated value $F(x, y)$ of $r(x, y)$ could be achieved, then,

$$
F(x, y)=r(x, y) \oplus R(x, y)
$$

Since red channel has the similar radiant distortion, $f_{R}(x, y)$ is used for the similarity of uniformity of illuminance so as to realize the correction.

$$
R_{G}(x, y)=\frac{r(x, y)}{f_{R}(x, y)} \times R(x, y) \times G(x, y) \times B(x, y)
$$

Where the constant $R_{G}(x, y)$ is used for adjusting the image brightness with the value $[0,1]$.

\section{Experimental Results and Discussions}

The experiment is designed to evaluate the proposed models for geometric and radiant correction. First of all, the distortion identification is carried out following the procedures:

1) Identify the models with high degree ( $>3$ ) according to the $N$ constraint points; 
2) Determine the parameter $\eta_{1}$ and $\eta_{2}$ using formula (7);

3) Determine the area increment difference $m=\lceil i\rceil$ and $n=\lceil j\rceil$, we can get $i$ and $j$.

4) Divide sub-areas using formula (17);

5) Identify each sub-area and work out the polynomial parameters.

The geometric correction proposed in this paper follows two major steps. First, the identified distorted model is used for coordinate change. Each pixel from the ideal image will be mapped in the distorted image with associated coordinate. Second, the gray value of the pixel is determined by using dual-linear interpolation method. This experiment compares the high polynomial geometric change algorithm ( 3 order polynomial algorithm) and a polynomial approximation slice model so as to test the efficiency and effectiveness.

\subsection{Geometric Correction Evaluation}

Figure 1 shows the experiment results. (a) is an average grid image with the size of $680 \times 720$ for doing the experiment. $w=480, h=640$. (b) and (c) are two types of captured images. (d)(e)(f) are the calibrated images using 3 order polynomial algorithm. $(\mathrm{g})(\mathrm{h})(\mathrm{i})$ are the calibrated results using the proposed method in this paper.

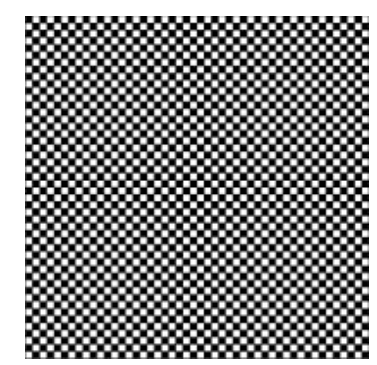

(a)

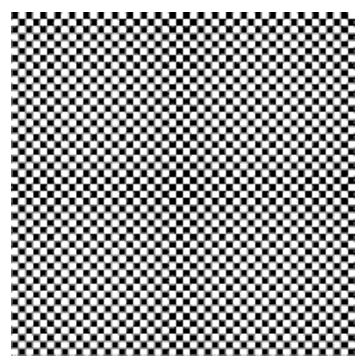

(d)

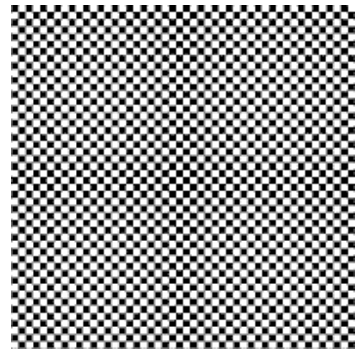

(g)

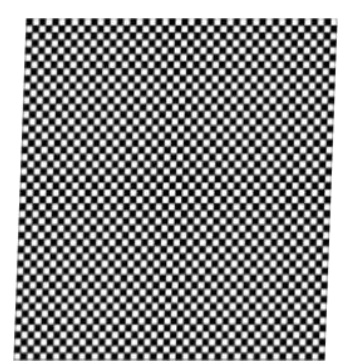

(b)

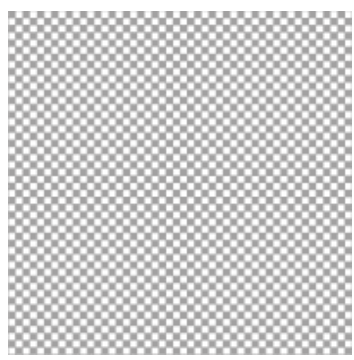

(e)

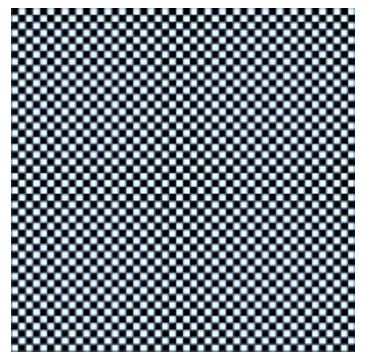

(h)

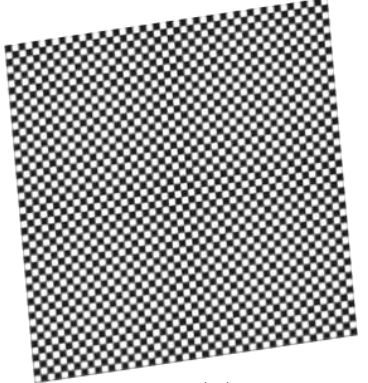

(c)

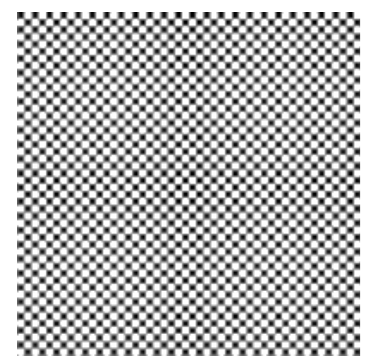

(f)

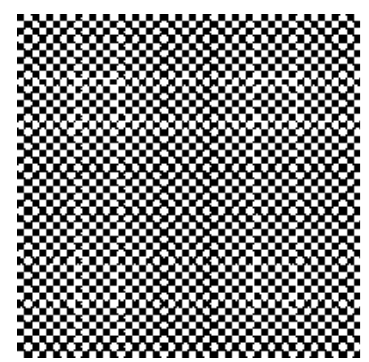

(i)

Figure 1. Geometric Correction Results 
From Figure 1, the proposed model outperforms the 3 order polynomial algorithm through the observation of effectiveness. We also compare the correction results quantitatively by calculating the pixel differences with central coordinate. Table 1 presents the comparison of different calibration methods with eight groups of coordinates.

Table 1. Comparison Results of Two Methods

\begin{tabular}{|c|c|c|c|}
\hline Coordinates & $\begin{array}{c}\text { 3 order polynomial } \\
\text { algorithm }\end{array}$ & Proposed Model & $\begin{array}{c}\text { Time Cost } \\
(\mathbf{m s})\end{array}$ \\
\hline 1 & $(-180,140)$ & $(-182,138)$ & 582 vs 190 \\
\hline 2 & $(-130,68)$ & $(-127,70)$ & 600 vs 192 \\
\hline 3 & $(-56,16)$ & $(-60,16)$ & 585 vs 195 \\
\hline 4 & $(0,230)$ & $(0,230)$ & 587 vs 189 \\
\hline 5 & $(0,-36)$ & $(0,-40)$ & 588 vs 193 \\
\hline 6 & $(58,-86)$ & $(60,-85)$ & 577 vs 198 \\
\hline 7 & $(94,-160)$ & $(94,-160)$ & 598 vs 201 \\
\hline 8 & $(220,-43)$ & $(221,-40)$ & 608 vs 207 \\
\hline
\end{tabular}

It is observed, from table 1, the correctness of proposed model slightly outperforms the 3 order polynomial algorithm considering the coordinates. From the time cost perspective, the proposed model spends much less computational time. From the eight group of experiments, 3 order polynomial algorithm averagely needs $590.6 \mathrm{~ms}$ for fulfilling the corrections. While, the proposed polynomial approximation slice model needs $195.6 \mathrm{~ms}$ averagely. Thus, the proposed model only requires one third of calculation time to reach the same purpose.

The second experiment uses the fundus image for testing the polynomial approximation slice model. Figure 2 presents the experiment results by using two fundus images. (a) and (c) are the initial fundus images. (b) and (d) are the calibrated images using the proposed model respectively.

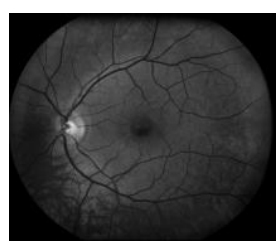

(a)

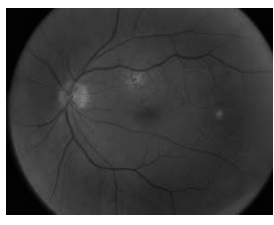

(c)

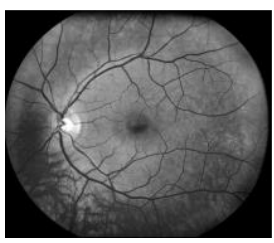

(b)

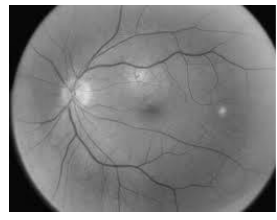

(d)

Figure 2. Fundus Image Results

Observed from Figure 2, the polynomial approximation slice model proposed in this paper has the ability to make full use of the advantage of polynomial coordinate change. The correction effectiveness is excellent compared with other algorithms. The gray fundus images could be operated by this model for addressing the geometric distortion. 


\subsection{Color Fundus Image Radiant Correction Experiment}

This experiment is going to evaluate the feasibility of the proposed color information radiant correction model. Four color fundus images are used for the testing. $R$ (red) and $G$ (green) channels are tested. Three other images are also tested such as view field of fundus image, calibrated fundus images, and comparison of histograms. Figure 3 presents the radiant distortion calibration results for colored fundus images.
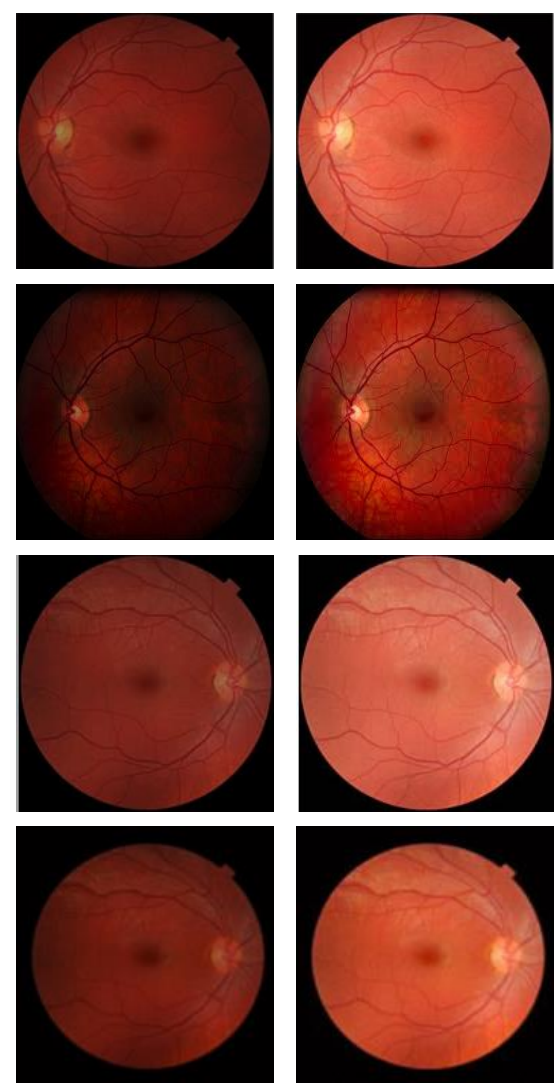

(a)

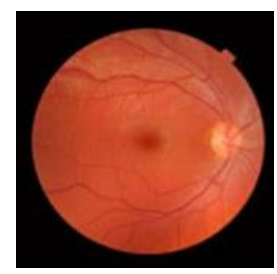

(b)
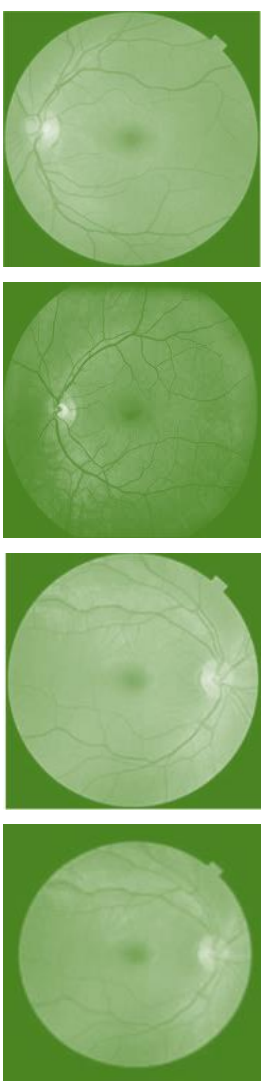

(c)
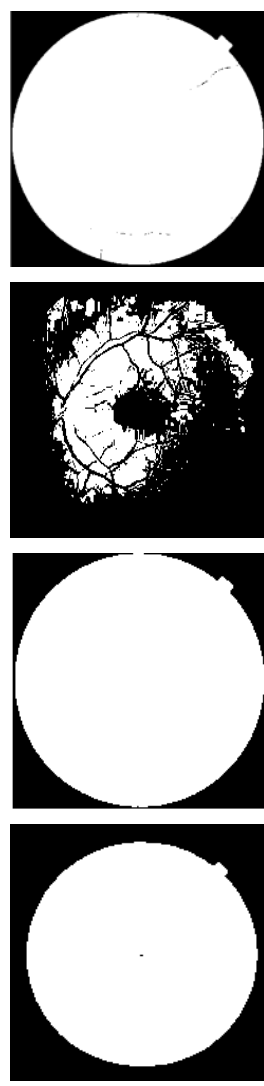

(d)
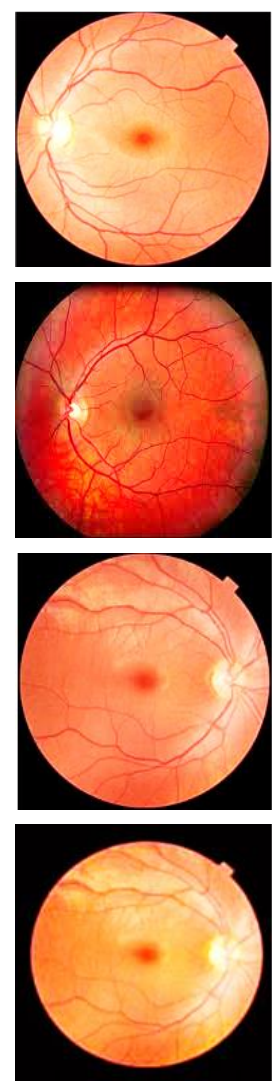

(e)

Figure 3. Radiant Distortion Calibration for Fundus Images

Figure 3 (a) are the initial fundus images for the experiments. (b) are the $R$ channel images. (c) are the $G$ channel images. (d) are the view field of fundus images. And (e) are the calibrated fundus images. From the above images, the images from $G$ channel are much darker than that from $R$ channel. The contract from $G$ channel is lower. After the correction processing by the proposed model, the brightness is significantly improved. The entire view field of fundus images has the average brightness. Meanwhile, the blood vessels' contracts have been greatly enhanced. Using the calibrated images, doctors are able to identify the diseases easily. Moreover, based on the correction, further processing like blood vessels extraction and characteristics presentations could be carried out.

The proposed algorithm uses the minimum storage space with the objective function of $\operatorname{Min} C(\alpha, \beta)$ which is able to divide the area into $\alpha \times \beta$ sub-areas. By using the Lagrange function, $\alpha$ and $\beta$ could be obtained thus, the algorithm is able to effectively and efficiently execute the image processing.

Figure 4 shows the histogram of the images from $G$ channel before and after the using of correction model so as to present the feasibility of the proposed model on 
image brightness and contract. $\mathrm{X}$-axis presents the brightness and $\mathrm{Y}$-axis presents the number of pixels.

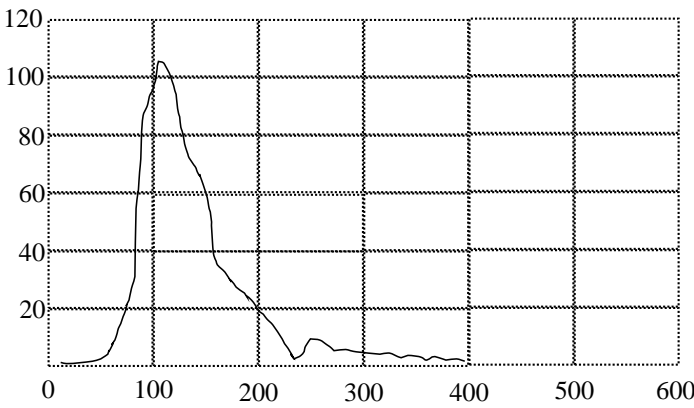

(a)

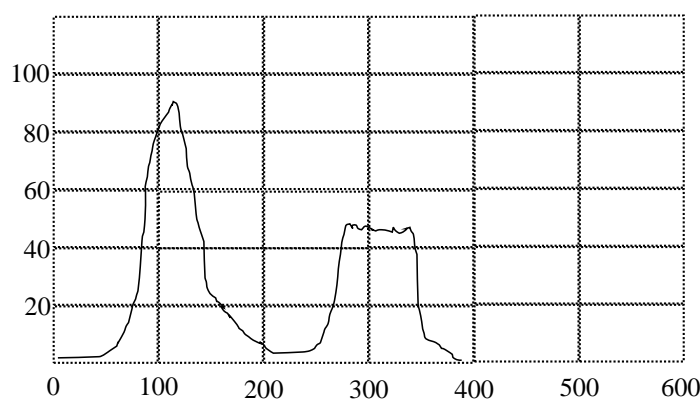

(c)

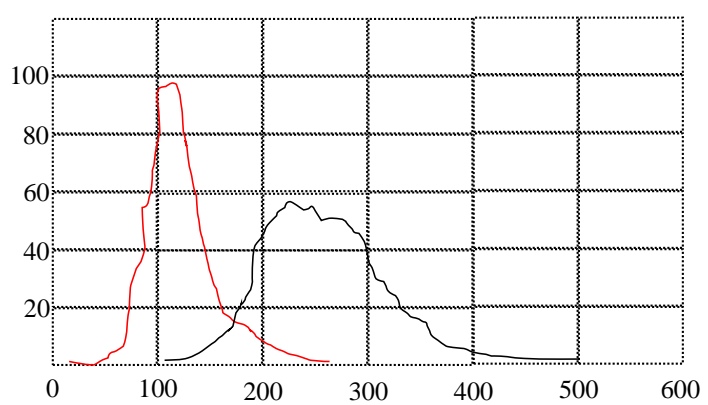

(b)

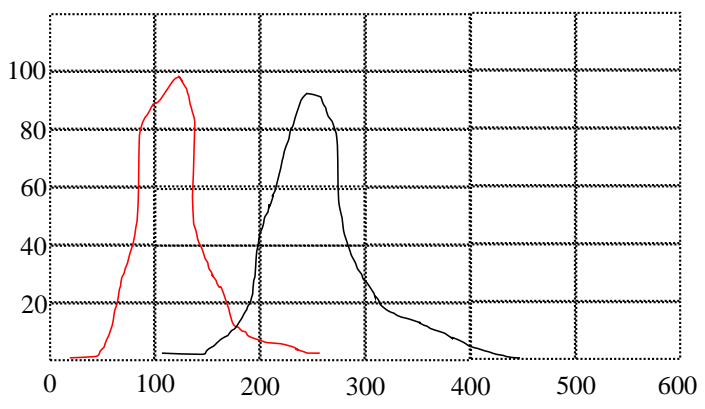

(d)

Figure 4. Comparison of Histograms

Figure 4 (a) and (c) present the histograms of the $G$ channel images from row 2 and 3. (b) and (d) show the calibrated images' histograms. It is observed that, after using the correction model, the curves become smoother. The total number of pixels are not changed significant, while the brightness is improved. The entire brightness becomes much clearer and the light area is extended. Therefore, the images' brightness balance and contracts are enhanced.

\section{Summary}

Eyes diseases are becoming more and more common since electronic devices are largely used in our daily life. Fundus images are then widely used to diagnose the eye diseases. This paper picks up the fundus images as research objects to propose an efficient and effective pre-processing model so as to deal with the image corrections. As most of the fundus images are captured or produced by the sophisticated equipment, image distortions are usually existing. This paper introduces a polynomial approximation slice model to address the geometric distortion and color information based radiant correction model to calibrate the captured fundus images. Experiments are designed to evaluate the feasibility and practicality of the proposed models. From the experimental results, it is found that after using the correction model, the total number of pixels are not changed significant, while the brightness is improved. The entire brightness becomes much clearer and the light area is extended.

Several conclusions and findings are significant as follows:

- From the eight group of experiments, 3 order polynomial algorithm averagely needs $590.6 \mathrm{~ms}$ for fulfilling the corrections. 
- the proposed polynomial approximation slice model needs $195.6 \mathrm{~ms}$ averagely.

- it is found that after using the correction model, the total number of pixels are not changed significant, while the brightness is improved.

Further studies are needed to improve this research. Firstly, this paper only considers the pre-processing of fundus images so as to deal with the distortions. Based on the correction, the segmentation of blood vessels and edge extractions will be studied in the near future. Therefore, for the diseased fundus images, it is possible to locate the diseased parts which will be helpful for diagnosing and solution making. Secondly, a prototype system using the proposed models designed and developed as services will be worked out. This system aims to assist the doctors to process the fundus images with improved correction and fast computational speed. As a result, the research outputs will be practically implemented in hospitals or clinics. Public society will be beneficial from this research hopefully in the future. Finally, the implementation of this research with proposed algorithm could be designed as an interface in real-life case and simulation studies.

\section{References}

[1] Z. Ghassabi, J. Shanbehzadeh, and A. Mohammadzadeh, "A structure-based region detector for highresolution retinal fundus image registration," Biomedical Signal Processing and Control, vol. 23, pp. 5261, (2016).

[2] F. Caliva, M. Aletti, B. Al-Diri, and A. Hunter, "A new tool to connect blood vessels in fundus retinal images," in Engineering in Medicine and Biology Society (EMBC), 2015 37 th Annual International Conference of the IEEE, pp. 4343-4346, (2015).

[3] R. Y. Zhong, Q. Y. Dai, T. Qu, G. J. Hu, and G. Q. Huang, "RFID-enabled Real-time Manufacturing Execution System for Mass-customization Production," Robotics and Computer-Integrated Manufacturing, vol. 29, pp. 283-292, (2013).

[4] R. Y. Zhong, G. Q. Huang, S. L. Lan, Q. Y. Dai, C. Xu, and T. Zhang, "A Big Data Approach for Logistics Trajectory Discovery from RFID-enabled Production Data," International Journal of Production Economics, vol. 165, pp. 260-272, (2015).

[5] R. Y. Zhong, S. Lan, C. Xu, Q. Dai, and G. Q. Huang, "Visualization of RFID-enabled shopfloor logistics Big Data in Cloud Manufacturing," The International Journal of Advanced Manufacturing Technology, vol. 84, pp. 5-16, (2016).

[6] C. Tsiotsios and M. Petrou, "On the choice of the parameters for anisotropic diffusion in image processing," Pattern recognition, vol. 46, pp. 1369-1381, (2013).

[7] Q. T. Nguyen, Y. G. Cao, G. W. Liu, N. P. Minh, and V. N. Le, "A Novel Approach for Shaken Image Deblurring," International Journal of Signal Processing, Image Processing and Pattern Recognition, vol. 7, pp. 85-98, (2014).

[8] M. Basha and B. Jinaga, "A New Approach Based on Order Reduction Using Sub Image Formation in Minimizing the Computation Time for Image Compression," International Journal of Signal Processing, Image Processing and Pattern Recognition, vol. 8, pp. 337-346, (2015).

[9] R. Y. Zhong, Z. Li, A. L. Y. Pang, Y. Pan, T. Qu, and G. Q. Huang, "RFID-enabled Real-time Advanced Planning and Scheduling Shell for Production Decision-making," International Journal of Computer Integrated Manufacturing. , vol. 26, pp. 649-662, (2013).

[10] S. R. Cho, Y. H. Park, G. P. Nam, K. Y. Shin, H. C. Lee, K. R. Park, S. M. Kim, and H. C. Kim, "Enhancement of Finger-Vein Image by Vein Line Tracking and Adaptive Gabor Filtering for FingerVein Recognition," Applied Mechanics and Materials, vol. 145, pp. 219-223, (2012).

[11] J. Odstrcilik, R. Kolar, A. Budai, J. Hornegger, J. Jan, J. Gazarek, et al., "Retinal vessel segmentation by improved matched filtering: evaluation on a new high-resolution fundus image database," Image Processing, IET, vol. 7, pp. 373-383, (2013).

[12] E. Trucco, A. Ruggeri, T. Karnowski, L. Giancardo, E. Chaum, J. P. Hubschman, et al., "Validating Retinal Fundus Image Analysis Algorithms: Issues and a ProposalValidating Retinal Fundus Image Analysis Algorithms," Investigative ophthalmology \& visual science, vol. 54, pp. 3546-3559, (2013).

[13] M. K. Dutta, M. Parthasarathi, S. Ganguly, S. Ganguly, and K. Srivastava, "An efficient image processing based technique for comprehensive detection and grading of nonproliferative diabetic retinopathy from fundus images," Computer Methods in Biomechanics and Biomedical Engineering: Imaging \& Visualization, pp. 1-13, (2015). 
[14] A. Singh, M. K. Dutta, M. ParthaSarathi, V. Uher, and R. Burget, "Image processing based automatic diagnosis of glaucoma using wavelet features of segmented optic disc from fundus image," Computer methods and programs in biomedicine, (2015).

[15] A. Kumar, A. K. Gaur, and M. Srivastava, "A segment based technique for detecting exudate from retinal fundus image," Procedia Technology, vol. 6, pp. 1-9, (2012).

[16] M. K. Dutta, K. Srivastava, S. Ganguly, S. Ganguly, M. Parthasarathi, R. Burget, et al., "Exudates detection in digital fundus image using edge based method \& strategic thresholding," 2015 38th International Conference on Telecommunications and Signal Processing (TSP), pp. 748-752, (2015).

[17] X. Tang, G. Zhang, X. Zhu, H. Pan, Y. Jiang, P. Zhou, et al., "Triple linear-array image geometry model of ZiYuan-3 surveying satellite and its validation," International Journal of Image and Data Fusion, vol. 4, pp. 33-51, (2013).

\section{Author}

Lanyan XUE, She graduated from Guangdong University of Technology. She is working as a lecturer in the Institute of Computer and Information, Fujian Agriculture and Forestry University, Fuzhou, China. Her research interests include pattern recognition, computer networks, and signal processing. 
International Journal of Signal Processing, Image Processing and Pattern Recognition Vol. 10, No. 2 (2017) 